\title{
Estimation of genetic merit from bivariate " all or none " responses
}

\author{
J.L. FOULLEY * and D. GIANOLA ** \\ * I.N.R.A., Station de Génétique quantitative et appliquée \\ Centre de Recherches zootechniques, F 78350 Jouy-en-Josas \\ ${ }^{* *}$ Department of Animal Science, University of Illinois \\ Urbana, Illinois 61801, U.S.A.
}

\begin{abstract}
Summary
A method of analysis of bivariate "all or none " categorical responses arising in animal breeding is presented. Conceptual bivariate normal variates following a mixed linear model are mapped onto a discrete scale via fixed thresholds. Parameters of the underlying scale are estimated in a Bayesian framework by finding the mode of a joint posterior distribution. The method requires iterative implementation and evaluation of bivariate normal integrals; estimation equations are presented. An application of the method to data on calf viability and calving ease is presented.
\end{abstract}

Key words : Multiple trait evaluation, all-or-none responses, Bayesian methods.

\begin{abstract}
Résumé
Estimation de la valeur génétique à partir de deux variables tout-ou-rien
\end{abstract}

Cet article présente une méthode d'analyse bidimensionnelle de caractères tout-ou-rien intervenant en sélection animale. La modélisation des réponses tout-ou-rien postule l'existence d'une distribution binormale de variables sous-jacentes à seuils qui suivent un modèle linéaire mixte. Les paramètres sur l'échelle sous-jacente sont estimés selon une procédure bayesienne à partir du mode de la distribution a posteriori. La méthode implique une résolution itérative et le calcul d'intégrales normales à 2 dimensions. Le système correspondant d'équations est décrit. La méthode est illustrée par une application à des données de viabilité et conditions de naissance de veaux.

Mots clés : Evaluation multicaractères, variables tout-ou-rien, méthode bayesienne.

\section{Introduction}

Categorical traits are ubiquitous in animal production. They play an important role as components of " numerical productivity", e.g., fertility, prolificacy, and viability. Variables of this type are often dealt with as if they were continuous, and analyzed via 
linear model methodology. Unfortunately, this approach is very difficult to justify because most hypotheses required to proceed with a linear analysis are clearly violated when the response variables are categorical (GIANOLA, 1982).

A general approach for prediction of genetic merit from categorical responses has been developed recently by GiANOLA \& Foulley $(1982,1983)$. This methodology relates discrete responses in mutually exclusive, exhaustive and ordered categories to conceptual underlying variates following a normal or a logistic distribution. The mapping from the conceptual to the discrete scale is done via a set of hypothetical successive thresholds which partition the real line into disjoint intervals. The underlying variates are modeled such that the sampling variability of levels of factors affecting the position of the distribution with respect to the thresholds is taken into account. This satisfies assumptions usually required in genetic analyses and gives considerable flexibility to the method, particularly when contrasted with others which accommodate a restricted set of models only (e.g., QUAAS \& VAN VLECK, 1980). The Bayesian framework of the procedure furnishes a conceptual liaison with mixed model prediction techniques for quantitative data (HENDERSON, 1973). This link becomes particularly clear in terms of the system of equations requiring solution : if the binomial or multinomial likelihood functions involved (GIANOLA \& Foulley, 1982, 1983) are replaced by a normal one, the method retrieves the "mixed model » equations of HENDERSON (1973).

An extension of the methodology to include mixtures of correlated normal and binary responses, with and without recursive relationships, was presented by FoulleY et al. (1983). The objective of this article is to extend the procedure to multiple categorical responses. For simplicity, 2 binary responses are considered, and an example in the domain of multiple trait evaluation for calving difficulty and calf viability is presented.

\section{Methodology}

\section{A. Data}

The data can be arranged as an $s \times 2 \times 2$ contingency table, where the $s$ rows represent combinations of levels of factors or, in its most extreme form, individuals themselves. The 2 nd and 3rd dimensions of the table correspond to 2 binary variates $(A, B)$, each with 2 mutually exclusive and exhaustive categories of response $[1,2]$. It is simpler to arrange the table as an $s \times 4$ array, with the 4 categories indicated as [11], [12], [21], [22]. For example, [12] denotes a response in the 1st category for variable $A$ and a response in the 2 nd category for variate $B$. Let $\mathrm{n}_{\mathrm{j}, \mathrm{k}}$ be the number of responses in category $\mathbf{k}(\mathrm{k}=11,12,21,22)$ within the $\mathrm{j}$ th row of the table (see table 1$), j=1, \ldots, s$. The marginal totals $n_{1}, \ldots, n_{j}, \ldots, n_{s .}$ are assumed fixed by sampling but can vary from row to row. The data are represented symbolically by the 4xs matrix :

$$
\mathbf{Y}=\left[\mathbf{Y}_{1}, \ldots, \mathbf{Y}_{\mathrm{j}}, \ldots, \mathbf{Y}_{\mathrm{s}}\right]
$$

where $\mathbf{Y}_{\mathrm{j}}$ is a $4 \times 1$ vector with :

$$
\mathbf{Y}_{\mathrm{j}}=\sum_{\mathrm{r}=1}^{\mathrm{n}_{\mathrm{j}} .} \mathbf{Y}_{\mathrm{jr}}
$$

and $\mathbf{Y}_{\mathrm{jr}}$ is a $4 \times 1$ vector having a 1 in the position corresponding to the category of response and zeroes elsewhere. 


\section{TABLE 1}

Bivariate binary responses arranged as an $s \times 4$ contingency table.

Données des 2 caractères tout-ou-rien présentées selon un tableau de contingence $s \times 4$.

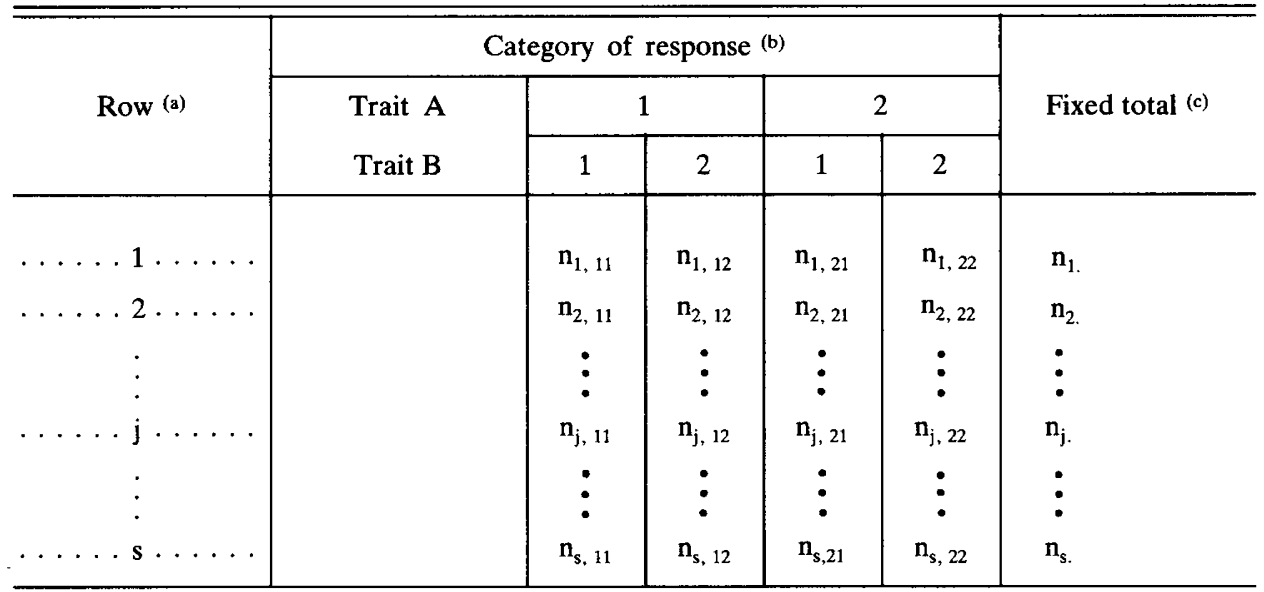

(a) Represents an individual or a combination of levels of explanatory variables.

(b) $\mathrm{n}_{\mathrm{j}, \mathrm{k}}$ is the number of counts in the $\mathrm{k}$ th category $(\mathrm{k}=11,12,21,22)$ within the $j$ th row.

(c) $\mathbf{n}_{\mathbf{j} .}=\mathbf{n}_{\mathbf{j}, 11}+\mathbf{n}_{\mathrm{j}, 12}+\mathbf{n}_{\mathrm{j}, 21}+\mathbf{n}_{\mathrm{j}, 22}$.

\section{B. Underlying model}

As in TAllis (1962), it is assumed that the probability that an experimental unit responds in the $\mathrm{k}$ th category is related to 2 conceptual variables, $\ell^{\mathrm{A}}$ and $\ell^{\mathrm{B}}$. Multivariate normality for these 2 variates can be justified if the hypothesis of multifactorial inheritance acting upon a non-negligible environmental background (the sum of many such effects) is tenable. The models for the 2 variables are :

$\ell_{j q}^{A}=\eta_{j}^{A}+\varepsilon_{j q}^{A}$

$\ell_{\mathrm{jq}}^{\mathrm{B}}=\eta_{\mathrm{j}}^{\mathrm{B}}+\varepsilon_{\mathrm{jq}}^{\mathrm{B}}$

where $\eta_{j q}^{A}$ and $\eta_{j}^{B}$ are location parameters, and $\varepsilon_{j q}^{A}$ and $\varepsilon_{j q}^{B}$ are residuals associated with the $q$ th experimental unit $\left(q=1, \ldots, n_{j}\right)$ in the $j$ th row of the table $(j=1, \ldots, s)$. The distribution of the residuals is :

$$
\left[\begin{array}{c}
\varepsilon_{\mathrm{jq}}^{\mathrm{A}} \\
\varepsilon_{\mathrm{jq}}^{\mathrm{B}}
\end{array}\right] \sim \mathrm{N}\left(\left[\begin{array}{l}
\mathrm{O} \\
\mathrm{O}
\end{array}\right],\left[\begin{array}{cc}
\sigma_{\mathrm{A}}^{2} & \rho \sigma_{\mathrm{A}} \sigma_{\mathrm{B}} \\
\varrho \sigma_{\mathrm{A}} \sigma_{\mathrm{B}} & \sigma_{\mathrm{B}}^{2}
\end{array}\right]\right)
$$

Given $\eta_{j}^{A}$ and $\eta_{j}^{B}$, the probability of response in a particular category $(11,12$, $21,22)$ is mapped via thresholds $t_{A}$ and $t_{B}$, such that :

$$
\begin{aligned}
& \text { Prob }\left\{11 \mid \eta_{j}^{A}, \eta_{j}^{B}\right\}=\operatorname{Prob}\left\{\ell_{A}<t_{A}, \ell_{B}<t_{B} \mid \eta_{j}^{A}, \eta_{j}^{B}\right\}=P_{j, 11} \\
& \text { Prob }\left\{12 \mid \eta_{j}^{A}, \eta_{j}^{B}\right\}=\operatorname{Prob}\left\{\ell_{A}<t_{A}, \ell_{B}>t_{B} \mid \eta_{j}^{A}, \eta_{j}^{B}\right\}=P_{j, 12}
\end{aligned}
$$




$$
\begin{aligned}
& \text { Prob }\left\{21 \mid \eta_{\mathrm{j}}^{\mathrm{A}}, \eta_{\mathrm{j}}^{\mathrm{B}}\right\}=\operatorname{Prob}\left\{\ell_{\mathrm{A}}>\mathrm{t}_{\mathrm{A}}, \ell_{\mathrm{B}}<\mathrm{t}_{\mathrm{B}} \mid \eta_{\mathrm{j}}^{\mathrm{A}}, \eta_{\mathrm{j}}^{\mathrm{B}}\right\}=\mathrm{P}_{\mathrm{j}, 21} \\
& \operatorname{Prob}\left\{22 \mid \eta_{\mathrm{j}}^{\mathrm{A}}, \eta_{\mathrm{j}}^{\mathrm{B}}\right\}=\operatorname{Prob}\left\{\ell_{\mathrm{A}}>\mathrm{t}_{\mathrm{A}}, \ell_{\mathrm{B}}>\mathrm{t}_{\mathrm{B}} \mid \eta_{\mathrm{j}}^{\mathrm{A}}, \eta_{\mathrm{j}}^{\mathrm{B}}\right\}=\mathrm{P}_{\mathrm{j}, 22}
\end{aligned}
$$

Now,

$$
P_{j, 11}=\int_{-\infty}^{t_{A}} \int_{-\infty}^{t_{B}} \phi(x, y) d x d y
$$

where $\phi(x, y)$ is a bivariate normal density function with means $\eta_{j}^{A}$ and $\eta_{j}^{B}$, and covariance matrix as in (5). Making the changes of variable :

$$
\mathrm{x}^{*}=\left(\mathrm{x}-\eta_{\mathrm{j}}^{\mathrm{A}}\right) / \sigma_{\mathrm{A}} ; \mathrm{y}^{*}=\left(\mathrm{y}-\eta_{\mathrm{j}}^{\mathrm{B}}\right) / \sigma_{\mathrm{B}}
$$

one can write (7) as :

$$
P_{j, 11}=\int_{-\infty}^{\mu_{i}^{A}} \int_{-\infty}^{\mu_{j}^{B}} \phi\left(x^{*}, y^{*} ; \varrho\right) d x^{*} d y^{*}=\Phi\left(\mu_{j}^{A}, \mu_{j}^{B} ; \varrho\right)
$$

where $\mu_{j}^{\mathrm{A}}=\left(\mathrm{t}_{\mathrm{A}}-\eta_{\mathrm{j}}^{\mathrm{A}}\right) / \sigma_{\mathrm{A}}$ and $\mu_{j}^{\mathrm{B}}=\left(\mathrm{t}_{\mathrm{B}}-\mu_{j}^{\mathrm{B}}\right) / \sigma_{\mathrm{B}}$, and $\phi(., . ; \varrho)$ and $\Phi(., . ; \varrho)$ are standard bivariate normal density and distribution functions, respectively, with a correlation coefficient $\varrho$. The following relationships hold :

$$
\begin{aligned}
& P_{j, 11}=\Phi\left(\mu_{j}^{\mathrm{A}}, \mu_{j}^{\mathrm{B}} ; \varrho\right) \\
& P_{j, 12}=\Phi\left(\mu_{\mathrm{j}}^{\mathrm{A}}\right)-\Phi\left(\mu_{\mathrm{j}}^{\mathrm{A}}, \mu_{\mathrm{j}}^{\mathrm{B}} ; \varrho\right) \\
& \mathrm{P}_{\mathrm{j}, 21}=\Phi\left(\mu_{\mathrm{j}}^{\mathrm{B}}\right)-\Phi\left(\mu_{\mathrm{j}}^{\mathrm{A}}, \mu_{\mathrm{j}}^{\mathrm{B}} ; \varrho\right) \\
& P_{\mathrm{j}, 22}=1-\Phi\left(\mu_{\mathrm{j}}^{\mathrm{A}}\right)-\Phi\left(\mu_{\mathrm{j}}^{\mathrm{B}}\right)+\Phi\left(\mu_{\mathrm{j}}^{\mathrm{A}}, \mu_{\mathrm{j}}^{\mathrm{B}} ; \varrho\right)
\end{aligned}
$$

where $\Phi($.$) is the standard normal distribution function. Let \boldsymbol{\mu}^{\mathrm{A}}=\left\{\boldsymbol{\mu}_{\mathrm{j}}^{\mathrm{A}}\right\}$ and $\boldsymbol{\mu}^{\mathrm{B}}=\left\{\mu_{j}^{\mathrm{B}}\right\}$ be $\mathrm{s} \times 1$ vectors with structure :

$$
\left[\begin{array}{l}
\boldsymbol{\mu}^{\mathrm{A}} \\
\boldsymbol{\mu}^{\mathrm{B}}
\end{array}\right]=\left[\begin{array}{ll}
\mathbf{X}_{\mathrm{A}} & \mathbf{0} \\
\mathbf{O} & \mathbf{X}_{\mathrm{B}}
\end{array}\right]\left[\begin{array}{l}
\boldsymbol{\beta}_{\mathrm{A}} \\
\boldsymbol{\beta}_{\mathrm{B}}
\end{array}\right]+\left[\begin{array}{ll}
\mathbf{Z}_{\mathrm{A}} & \mathbf{0} \\
\mathbf{O} & \mathbf{Z}_{\mathrm{B}}
\end{array}\right]\left[\begin{array}{l}
\mathbf{u}_{\mathrm{A}} \\
\mathbf{u}_{\mathrm{B}}
\end{array}\right]
$$

where $\mathbf{X}_{A}\left(\mathbf{X}_{B}\right)$ and $\mathbf{Z}_{A}\left(\mathbf{Z}_{\mathrm{B}}\right)$ are known matrices relating $\boldsymbol{\mu}^{\mathrm{A}}\left(\boldsymbol{\mu}^{\mathrm{B}}\right)$ to $\boldsymbol{\beta}_{\mathrm{A}}\left(\boldsymbol{\beta}_{\mathrm{B}}\right)$ and to $\mathbf{u}_{\mathrm{A}}\left(\mathbf{u}_{\mathrm{B}}\right)$, respectively. In particular,

$$
\begin{aligned}
& \mu_{\mathrm{j}}^{\mathrm{A}}=\mathbf{x}_{\mathrm{jA}}^{\prime} \boldsymbol{\beta}_{\mathrm{A}}+\mathbf{z}_{\mathrm{jA}}^{\prime} \mathbf{u}_{\mathrm{A}} \\
& \mu_{\mathrm{j}}^{\mathrm{B}}=\mathbf{x}_{\mathrm{j} B}^{\prime} \boldsymbol{\beta}_{\mathrm{B}}+\mathbf{z}_{\mathrm{j} B}^{\prime} \mathbf{u}_{\mathrm{B}}
\end{aligned}
$$

where $\mathbf{x}_{\mathrm{jA}}^{\prime}\left(\mathbf{x}_{\mathrm{jB}}^{\prime}\right)$ is the $\mathrm{j}$ th row of $\mathbf{X}_{\mathrm{A}}\left(\mathbf{X}_{\mathrm{B}}\right)$, and $\mathbf{z}_{\mathrm{jA}}^{\prime}\left(\mathbf{z}_{\mathrm{jB}}^{\prime}\right)$ is the $\mathrm{j}$ th row of $\mathbf{Z}_{\mathrm{A}}\left(\mathbf{Z}_{\mathrm{B}}\right)$. More generally, (11) can be written as :

$$
\boldsymbol{\mu}=\mathbf{X} \boldsymbol{\beta}+\mathbf{Z u}
$$

where, without loss of generality, $\mathbf{X}$ can be taken as a full-rank matrix.

\section{Inference}

With $\boldsymbol{\theta}^{\prime}=\left[\boldsymbol{\beta}^{\prime}, \mathbf{u}^{\prime}\right]$, inferences are based on Bayes theorem (e.g., LiNDLEY, 1965) :

$$
\mathrm{f}(\boldsymbol{\theta} \mid \mathbf{Y}) \propto \mathrm{g}(\mathbf{Y} \mid \boldsymbol{\theta}) . \mathrm{h}(\boldsymbol{\theta})
$$


where $f(\boldsymbol{\theta} \mid \mathbf{Y})$ is the posterior density, $g(\mathbf{Y} \mid \boldsymbol{\theta})$ is the likelihood function and $\mathrm{h}(\boldsymbol{\theta})$ is the prior density.

A priori, we take :

$$
\left[\begin{array}{l}
\boldsymbol{\beta} \\
\mathbf{u}
\end{array}\right] \sim \mathbf{N}\left(\left[\begin{array}{l}
\boldsymbol{\alpha} \\
\boldsymbol{O}
\end{array}\right],\left[\begin{array}{ll}
\boldsymbol{\Gamma} & \mathbf{O} \\
\mathbf{O} & \mathbf{G}
\end{array}\right]\right)
$$

where :

$$
\begin{gathered}
\boldsymbol{\alpha}^{\prime}=\left[\boldsymbol{\alpha}_{\mathrm{A}}^{\prime}, \boldsymbol{\alpha}_{\mathrm{B}}^{\prime}\right] \\
\boldsymbol{\Gamma}=\left[\begin{array}{ll}
\Gamma_{\mathrm{AA}} & \Gamma_{\mathrm{AB}} \\
\Gamma_{\mathrm{BA}} & \Gamma_{\mathrm{BB}}
\end{array}\right] \\
\mathbf{G}=\left[\begin{array}{ll}
\mathbf{G}_{\mathrm{AA}} & \mathbf{G}_{\mathrm{AB}} \\
\mathbf{G}_{\mathrm{BA}} & \mathbf{G}_{\mathrm{BB}}
\end{array}\right]
\end{gathered}
$$

In genetic applications, $\mathbf{u}^{\prime}=\left[\mathbf{u}_{\mathrm{A}}^{\prime}, \mathbf{u}_{\mathrm{B}}^{\prime}\right]$ is usually a vector of additive genetic values or of "transmitting abilities", in which case $\mathbf{G}$ is a function of the additive relationship matrix between individuals and of the genetic variances and covariances for traits $A$ and $B$. For example, when $\mathbf{u}_{A}$ and $\mathbf{u}_{B}$ are vectors of additive genetic values to be predicted in the same individuals :

$$
\mathbf{G}_{\mathrm{AA}}=\mathbf{A} \sigma_{\mathrm{u}_{\mathrm{A}}}^{2} ; \mathbf{G}_{\mathrm{AB}}=\mathbf{A} \sigma_{\mathrm{u}_{\mathrm{AB}}} ; \mathbf{G}_{\mathrm{BB}}=\mathbf{A} \sigma_{\mathrm{u}_{\mathrm{B}}}^{2}
$$

where $A$ is the additive relationship matrix, $\sigma_{u_{A}}^{2}\left(\sigma_{u_{B}}^{2}\right)$ is the additive genetic variance of trait $A(B)$, and $\sigma_{u_{A B}}$ is the additive genetic covariance between traits $A$ and $B$. Apart from a proportionality factor, the prior density is then from (15) to (18) :

$$
\mathrm{f}(\boldsymbol{\theta}) \propto \exp \left\{-\frac{1}{2}\left[(\boldsymbol{\beta}-\boldsymbol{\alpha})^{\prime} \boldsymbol{\Gamma}^{-1}(\boldsymbol{\beta}-\boldsymbol{\alpha})+\mathbf{u}^{\prime} \mathbf{G}^{-1} \mathbf{u}\right]\right\}
$$

Given $\boldsymbol{\theta}$, the vectors $\mathbf{Y}_{\mathrm{j}}$ in (2) are conditionally independent and the likelihood function is then :

$$
g(\mathbf{Y} \mid \boldsymbol{\theta})=\prod_{j=1}^{s}\left\{\frac{n_{j, !}}{\prod_{k} n_{j, k !}} \prod_{k} P_{j, k}^{n_{j, k}}\right\}
$$

The posterior density in (14) is then proportional to the product of (20) and (21). The selection rule which maximizes the expected merit of a fixed number of selected candidates (GOFFINET, 1983) is based on :

$$
\mathrm{E}(\boldsymbol{\theta} \mid \mathbf{Y})=\int_{\mathbb{R}^{\mathrm{d}}} \boldsymbol{\gamma f}(\boldsymbol{\gamma} \mid \mathbf{Y}) \mathrm{d} \boldsymbol{\gamma} \text { where } \mathrm{d} \text { is the order of } \boldsymbol{\theta} .
$$

$\mathrm{E}(\boldsymbol{\theta} \mid \mathbf{Y})$ is called the posterior mean. Unfortunately (22) is technically impossible to evaluate. In the present paper, we calculate the posterior mode and regard it as an approximation to $E(\boldsymbol{\theta} \mid \mathbf{Y})$. If the posterior density is symmetric and unimodal, $E(\boldsymbol{\theta} \mid \mathbf{Y})$ and the posterior mode are identical. Otherwise, as $\mathbf{n}_{\mathrm{j}}$. increases, the likelihood function and the posterior density become normal so, in the limit, the posterior mean and mode 
become the same. Alternatively, the posterior mode corresponds to a Bayesian estimator which minimizes the expected posterior loss when the loss function is :

$$
\sum_{i} d_{i} \text { with } d_{i}\left\{\begin{array}{l}
1, \text { for }\left|\theta_{i}-\theta_{i}\right|>\varepsilon \\
0, \text { otherwise }
\end{array}\right.
$$

where $\varepsilon$ is an arbitrarily small positive number (PRATT et al., 1965).

\section{Computations}

\section{A. Principles}

The log-posterior density can be written from (14), (20) and (21) as :

$$
\begin{gathered}
\mathrm{L}(\boldsymbol{\theta})=\mathrm{C}+\sum_{\mathrm{j}} \sum_{\mathrm{k}} \mathrm{n}_{\mathrm{j}, \mathrm{k}} \ln \left(\mathrm{P}_{\mathrm{j}, \mathrm{k}}\right) \\
-\frac{1}{2}\left[(\boldsymbol{\beta}-\boldsymbol{\alpha})^{\prime} \boldsymbol{\Gamma}^{-1}(\boldsymbol{\beta}-\boldsymbol{\alpha})+\mathbf{u}^{\prime} \mathbf{G}^{-1} \mathbf{u}\right]
\end{gathered}
$$

where $\mathrm{C}$ is a constant. In this paper we assume that prior knowledge about $\boldsymbol{\beta}$ is vague, i.e., $\Gamma^{-1} \rightarrow 0$, so we can write :

$$
\mathrm{L}(\boldsymbol{\theta})=\sum_{\mathrm{j}} \sum_{\mathrm{k}} \mathrm{n}_{\mathrm{j}, \mathrm{k}} \ln \left(\mathrm{P}_{\mathrm{j}, \mathrm{k}}\right)-\frac{1}{2} \mathbf{u}^{\prime} \mathbf{G}^{-1} \mathbf{u}+\mathrm{C}
$$

Finding the mode requires solving :

$$
\frac{\partial \mathrm{L}(\boldsymbol{\theta})}{\partial \boldsymbol{\theta}}=0
$$

which in this case yields a non-linear system ; therefore, an iterative solution is required. We use the method of Newton-Raphson (DAHLQUIST \& BJORCK, 1974) which consists in iterating with :

$$
-\left[\frac{\partial^{2} \mathrm{~L}(\boldsymbol{\theta})}{\partial \boldsymbol{\theta} \partial \boldsymbol{\theta}^{\prime}}\right]_{\boldsymbol{\theta}=\hat{\boldsymbol{\theta}}[\mathrm{i}-1]} \Delta^{[\mathrm{i}]}=\left[\frac{\partial \mathrm{L}(\boldsymbol{\theta})}{\partial \theta}\right]_{\boldsymbol{\theta}=\hat{\theta}}[\mathrm{i}-1]
$$

where $\Delta^{[i]}=\hat{\boldsymbol{\theta}}^{[\mathrm{i}]}-\hat{\boldsymbol{\theta}}^{[\mathrm{i}-1]}$, and $\hat{\boldsymbol{\theta}}^{[\mathrm{i}]}$ is a solution at the ith iterate. Iteration stops when $\Delta[i]<\epsilon$, the latter being a vector of arbitrarily small numbers.

\section{B. Derivatives}

As shown in Annex $\mathrm{A}$, the first derivatives are :

$$
\begin{aligned}
& \frac{\partial L(\boldsymbol{\theta})}{\partial \boldsymbol{\beta}_{\mathrm{A}}}=\mathbf{X}_{\mathrm{A}}^{\prime} \mathbf{v}_{\mathrm{A}} \\
& \frac{\partial \mathrm{L}(\boldsymbol{\theta})}{\partial \boldsymbol{\beta}_{\mathrm{B}}}=\mathbf{X}_{\mathrm{B}}^{\prime} \mathbf{v}_{\mathrm{B}}
\end{aligned}
$$




$$
\begin{aligned}
& \frac{\partial L(\theta)}{\partial \mathbf{u}_{A}}=\mathbf{Z}_{A}^{\prime} \mathbf{v}_{A}-G^{A A} \mathbf{u}_{A}-G^{A B} \mathbf{u}_{B} \\
& \frac{\partial L(\theta)}{\partial \mathbf{u}_{B}}=\mathbf{Z}_{B}^{\prime} \mathbf{v}_{B}-G^{B A} \mathbf{u}_{A}-G^{B B} \mathbf{u}_{B}
\end{aligned}
$$

where :

$$
\left[\begin{array}{ll}
\mathbf{G}^{\mathrm{AA}} & \mathbf{G}^{\mathrm{AB}} \\
\mathbf{G}^{\mathrm{BA}} & \mathbf{G}^{\mathrm{BB}}
\end{array}\right]=\left[\begin{array}{ll}
\mathbf{G}_{\mathrm{AA}} & \mathbf{G}_{\mathrm{AB}} \\
\mathbf{G}_{\mathrm{BA}} & \mathbf{G}_{\mathrm{BB}}
\end{array}\right]^{-1}
$$

and $\mathbf{v}_{A}$ and $\mathbf{v}_{B}$ as shown in the Annex.

It is shown in Annex B that the second derivatives can be written as :

$$
\begin{aligned}
& \frac{\partial^{2} \mathbf{L}(\boldsymbol{\theta})}{\partial \boldsymbol{\beta}_{\mathrm{A}} \partial \boldsymbol{\beta}_{\mathrm{A}}^{\prime}}=-\mathbf{X}_{\mathrm{A}}^{\prime} \mathbf{W}_{\mathrm{AA}} \mathbf{X}_{\mathrm{A}} \\
& \frac{\partial^{2} \mathrm{~L}(\boldsymbol{\theta})}{\partial \boldsymbol{\beta}_{\mathrm{A}} \partial \boldsymbol{\beta}_{\mathrm{B}}^{\prime}}=-\mathbf{X}_{\mathrm{A}}^{\prime} \mathbf{W}_{\mathrm{AB}} \mathbf{X}_{\mathrm{B}} \\
& \frac{\partial^{2} \mathbf{L}(\boldsymbol{\theta})}{\partial \boldsymbol{\beta}_{\mathrm{A}} \partial \mathbf{u}_{\mathrm{A}}^{\prime}}=-\mathbf{X}_{\mathrm{A}}^{\prime} \mathbf{W}_{\mathrm{AA}} \mathbf{Z}_{\mathrm{A}} \\
& \frac{\partial^{2} \mathbf{L}(\boldsymbol{\theta})}{\partial \boldsymbol{\beta}_{\mathrm{A}} \partial \mathbf{u}_{\mathrm{B}}^{\prime}}=-\mathbf{X}_{\mathrm{A}}^{\prime} \mathbf{W}_{\mathrm{AB}} \mathbf{Z}_{\mathrm{B}} \\
& \frac{\partial^{2} \mathrm{~L}(\boldsymbol{\theta})}{\partial \boldsymbol{\beta}_{\mathrm{B}} \partial \boldsymbol{\beta}_{\mathrm{B}}^{\prime}}=-\mathbf{X}_{\mathrm{B}}^{\prime} \mathbf{W}_{\mathrm{BB}} \mathbf{X}_{\mathrm{B}} \\
& \frac{\partial^{2} \mathbf{L}(\boldsymbol{\theta})}{\partial \boldsymbol{\beta}_{\mathrm{B}} \partial \mathbf{u}_{\mathrm{A}}^{\prime}}=-\mathbf{X}_{\mathrm{B}}^{\prime} \mathbf{W}_{\mathrm{BA}} \mathbf{Z}_{\mathrm{A}} \\
& \frac{\partial^{2} \mathbf{L}(\boldsymbol{\theta})}{\partial \boldsymbol{\beta}_{\mathrm{B}} \partial \mathbf{u}_{\mathrm{B}}^{\prime}}=-\mathbf{X}_{\mathrm{B}}^{\prime} \mathbf{W}_{\mathrm{BB}} \mathbf{Z}_{\mathrm{B}} \\
& \frac{\partial^{2} \mathbf{L}(\boldsymbol{\theta})}{\partial \mathbf{u}_{\mathrm{A}} \partial \mathbf{u}_{\mathrm{A}}^{\prime}}=-\mathbf{Z}_{\mathrm{A}}^{\prime} \mathbf{W}_{\mathrm{AA}} \mathbf{Z}_{\mathrm{A}}-\mathbf{G}^{\mathrm{AA}} \\
& \frac{\partial^{2} \mathbf{L}(\boldsymbol{\theta})}{\partial \mathbf{u}_{\mathrm{A}} \partial \mathbf{u}_{\mathrm{B}}}=-\mathbf{Z}_{\mathrm{A}}^{\prime} \mathbf{W}_{\mathrm{AB}} \mathbf{Z}_{\mathrm{B}}-\mathbf{G}^{\mathrm{AB}} \\
& \frac{\partial^{2} \mathbf{L}(\boldsymbol{\theta})}{\partial \mathbf{u}_{\mathrm{B}} \partial \mathbf{u}_{\mathrm{B}}^{\prime}}=-\mathbf{Z}_{\mathrm{B}}^{\prime} \mathbf{W}_{\mathrm{BB}} \mathbf{Z}_{\mathrm{B}}-\mathbf{G}^{\mathrm{BB}}
\end{aligned}
$$

where $\mathbf{w}_{\mathrm{AA}}, \mathbf{W}_{\mathrm{BB}}$ and $\mathbf{w}_{\mathrm{AB}}$ are diagonal matrices, with $\mathbf{w}_{\mathrm{AB}}=\mathbf{w}_{\mathrm{BA}}$. 


\section{Estimation equations}

Using (26 a-d) and (28 a-j) in (25) yields the system :

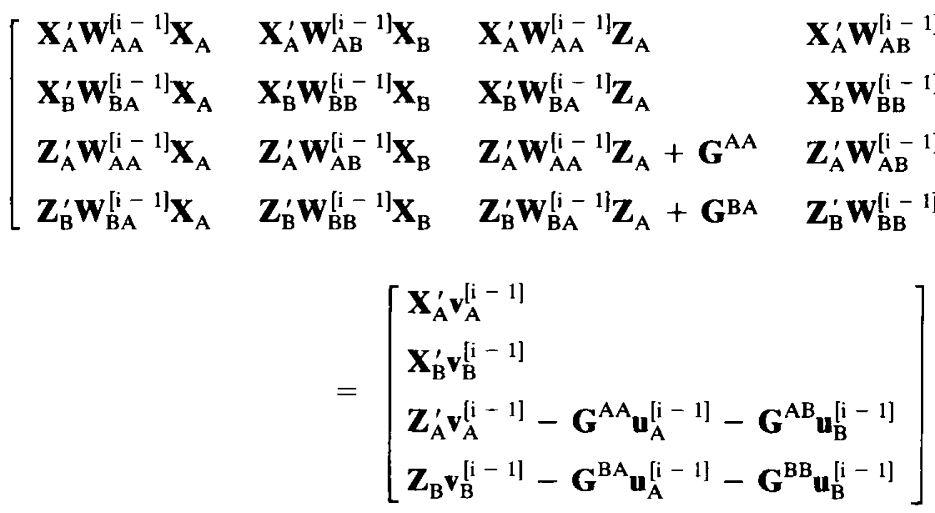

where the brackets indicate the round of iteration, and :

$$
\begin{aligned}
& \boldsymbol{\Delta}_{\beta_{A}}^{[i]}=\beta_{A}^{[i]}-\beta_{A}^{[i-1]}, \Delta_{\beta_{B}}^{[i]}=\beta_{B}^{[i]}-\beta_{B}^{[i-1]}, \\
& \Delta_{u_{A}}^{[i]}=\mathbf{u}_{A}^{[i]}-\mathbf{u}_{A}^{[i-1]}, \text { and } \Delta_{u_{B}}^{[i]}=\mathbf{u}_{B}^{[i]}-\mathbf{u}_{B}^{[i-1]} .
\end{aligned}
$$

Equations (29) can be rewritten as :

$$
\begin{aligned}
& {\left[\begin{array}{llll}
\mathbf{X}_{\mathrm{A}}^{\prime} \mathbf{W}_{\mathrm{AA}}^{[\mathrm{i}-1]} \mathbf{X}_{\mathrm{A}} & \mathbf{X}_{\mathrm{A}}^{\prime} \mathbf{W}_{\mathrm{AB}}^{[\mathrm{i}-1]} \mathbf{X}_{\mathrm{B}} & \mathbf{X}_{\mathrm{A}}^{\prime} \mathbf{W}_{\mathrm{AA}}^{[\mathrm{i}-1]} \mathbf{Z}_{\mathrm{A}} & \mathbf{X}_{\mathrm{A}}^{\prime} \mathbf{W}_{\mathrm{AB}}^{[\mathrm{i}-1]} \mathbf{Z}_{\mathrm{B}} \\
\mathbf{X}_{\mathrm{B}}^{\prime} \mathbf{W}_{\mathrm{BA}}^{[\mathrm{i}-1]} \mathbf{X}_{\mathrm{A}} & \mathbf{X}_{\mathrm{B}}^{\prime} \mathbf{W}_{\mathrm{BB}}^{[\mathrm{i}-1]} \mathbf{X}_{\mathrm{B}} & \mathbf{X}_{\mathrm{B}}^{\prime} \mathbf{W}_{\mathrm{BA}}^{[\mathrm{i}-1]} \mathbf{Z}_{\mathrm{A}} & \mathbf{X}_{\mathrm{B}}^{\prime} \mathbf{W}_{\mathrm{BB}}^{[\mathrm{i}-1]} \mathbf{Z}_{\mathrm{B}} \\
\mathbf{Z}_{\mathrm{A}}^{\prime} \mathbf{W}_{\mathrm{AA}}^{[\mathrm{i}-1]} \mathbf{X}_{\mathrm{A}} & \mathbf{Z}_{\mathrm{A}}^{\prime} \mathbf{W}_{\mathrm{AB}}^{[\mathrm{i}-1]} \mathbf{X}_{\mathrm{B}} & \mathbf{Z}_{\mathrm{A}}^{\prime} \mathbf{W}_{\mathrm{AA}}^{[\mathrm{i}-1]} \mathbf{Z}_{\mathrm{A}}+\mathbf{G}^{\mathrm{AA}} & \mathbf{Z}_{\mathrm{A}}^{\prime} \mathbf{W}_{\mathrm{AB}}^{[\mathrm{i}-1]} \mathbf{Z}_{\mathrm{B}}+\mathbf{G}^{\mathrm{AB}} \\
\mathbf{Z}_{\mathrm{B}}^{\prime} \mathbf{W}_{\mathrm{BA}}^{[\mathrm{i}-1]} \mathbf{X}_{\mathrm{A}} & \mathbf{Z}_{\mathrm{B}}^{\prime} \mathbf{W}_{\mathrm{BB}}^{[\mathrm{i}-1]} \mathbf{X}_{\mathrm{B}} & \mathbf{Z}_{\mathrm{B}}^{\prime} \mathbf{W}_{\mathrm{BA}}^{[\mathrm{i}-1]} \mathbf{Z}_{\mathrm{A}}+\mathbf{G}^{\mathrm{BA}} & \mathbf{Z}_{\mathrm{B}}^{\prime} \mathbf{W}_{\mathrm{BB}}^{[\mathrm{i}-1]} \mathbf{Z}_{\mathrm{B}}+\mathbf{G}^{\mathrm{BB}}
\end{array}\right]\left[\begin{array}{c}
\boldsymbol{\beta}_{\mathrm{A}}^{[\mathrm{ij}]} \\
\boldsymbol{\beta}_{\mathrm{B}}^{[\mathrm{i}]} \\
\mathbf{u}_{\mathrm{A}}^{[\mathrm{i}]} \\
\mathbf{u}_{\mathrm{B}}^{[\mathrm{i}]}
\end{array}\right]} \\
& =\left[\begin{array}{l}
\mathbf{X}_{A}^{\prime} \mathbf{W}_{A A}^{[i-1]} \mathbf{y}_{A}^{[i-1]}+\mathbf{X}_{A}^{\prime} \mathbf{W}_{A B}^{[i-1]} \mathbf{y}_{B}^{[i-1]} \\
\mathbf{X}_{B}^{\prime} \mathbf{W}_{B B}^{[i-1]} \mathbf{y}_{B}^{[i-1]}+\mathbf{X}_{B}^{\prime} \mathbf{W}_{B A}^{[i-1]} \mathbf{y}_{A}^{[i-1]} \\
\mathbf{Z}_{A}^{\prime} \mathbf{W}_{A A}^{[i-1]} \mathbf{y}_{A}^{[i-1]}+\mathbf{Z}_{A}^{\prime} \mathbf{W}_{A B}^{[i-1]} \mathbf{y}_{B}^{[i-1]} \\
\mathbf{Z}_{B}^{\prime} \mathbf{W}_{B B}^{[i-1]} \mathbf{y}_{B}^{[i-1]}+\mathbf{Z}_{B}^{\prime} \mathbf{W}_{B A}^{[i-1]} \mathbf{y}_{A}^{[i-1]}
\end{array}\right]
\end{aligned}
$$

where :

$$
\left[\begin{array}{c}
\mathbf{y}_{A}^{[i]} \\
\mathbf{y}_{B}^{[i]}
\end{array}\right]=\left[\begin{array}{c}
\mathbf{X}_{A} \beta_{A}^{[i]}+\mathbf{Z}_{A} \mathbf{u}_{A}^{[i]} \\
\mathbf{X}_{B} \beta_{B}^{[i]}+\mathbf{Z}_{B} \mathbf{u}_{B}^{[i]}
\end{array}\right]+\left[\begin{array}{cc}
\mathbf{W}_{A A}^{[i]} & \mathbf{W}_{A B}^{[i]} \\
\mathbf{W}_{B A}^{[i]} & \mathbf{W}_{B B}^{[i]}
\end{array}\right]-1\left[\begin{array}{c}
\mathbf{v}_{A}^{[i]} \\
\mathbf{v}_{B}^{[i]}
\end{array}\right]
$$

The parallel between (30) and the multiple trait " mixed model " equations (e.g., HENDERSON \& QUAAS, 1976 ; Foulley et al., 1982) is remarkable. With normal data, the $\mathbf{W}$ matrices are segments of the inverse of the residual variance-covariance matrix, and the "working" variates $\left(\mathbf{y}_{\mathrm{A}}, \mathbf{y}_{\mathrm{B}}\right)$ in (31) are replaced by observations in traits $\mathrm{A}$ and $\mathrm{B}$. 


\section{Numerical example}

\section{A. Data}

We consider a hypothetical example discussed by ScHAEFFER \& WiLTON (1976). These authors gave data for calf viability and for a subjective assessment of degree of calving difficulty. Response categories for calf viability were " alive " or " dead ". Those for calving difficulty were " normal" or " assisted"; these resulted by regrouping SCHAEFFER \& WiltON's (1976) 3 classes into 2 : normal (or not observed) vs. light or extreme difficulty. The data comprised 28 calving records classified by herd-year ( 2 levels), calf's dam age (heifers vs. cows), calf sex (male vs. female) and sire of calf (4 levels). The records were arranged as a $20 \times 4$ contingency table as shown in table 2 . The rows consisted of 20 combinations of herd-year $\times$ age of dam $\times$ sex of calf $\times$ sire subclasses ; the 4 columns were [11] (alive, normal birth), [12] (alive, assisted birth), [21] (dead, normal birth) and [22] (dead, assisted birth).

TABLE 2

Distribution of calving records by herd-year, age of dam, sex and sire of calf subclasses (SCHAEFFER \& WILTON, 1976).

Répartition des données de vêlage selon le troupeau $x$ année, l'âge de la mère, le sexe et le père du veau (SCHAEFFER \& WILTON, 1976).

\begin{tabular}{c|c|c|c|c|c|c|c|c}
\hline \hline \multirow{2}{*}{ Row } & \multirow{2}{*}{$\begin{array}{c}\text { Herd- } \\
\text { Year }\end{array}$} & \multirow{2}{*}{$\begin{array}{c}\text { Dam's } \\
\text { Age }\end{array}$} & $\begin{array}{c}\text { Calf's } \\
\text { Sex (a) }\end{array}$ & $\begin{array}{c}\text { Calf's } \\
\text { Sire }\end{array}$ & \multicolumn{4}{|c|}{ Counts in category (b) } \\
\cline { 5 - 8 } & & & & {$[11]$} & {$[12]$} & {$[21]$} & {$[22]$} \\
\hline & & & & & & & & \\
1 & 1 & 2 & M & 1 & 1 & 0 & 0 & 0 \\
2 & 1 & 2 & F & 1 & 1 & 0 & 0 & 0 \\
3 & 1 & 3 & M & 1 & 0 & 0 & 1 & 0 \\
4 & 1 & 2 & F & 2 & 0 & 1 & 0 & 0 \\
5 & 1 & 3 & M & 2 & 1 & 1 & 0 & 0 \\
6 & 1 & 3 & F & 2 & 2 & 0 & 1 & 0 \\
7 & 1 & 2 & M & 3 & 1 & 0 & 0 & 1 \\
8 & 1 & 3 & F & 3 & 0 & 0 & 0 & 1 \\
9 & 1 & 3 & M & 3 & 1 & 0 & 0 & 0 \\
10 & 2 & 2 & F & 1 & 2 & 0 & 0 & 0 \\
11 & 2 & 2 & M & 1 & 1 & 0 & 0 & 0 \\
12 & 2 & 3 & M & 1 & 0 & 0 & 0 & 1 \\
13 & 2 & 2 & F & 2 & 1 & 0 & 0 & 1 \\
14 & 2 & 3 & M & 2 & 1 & 0 & 0 & 0 \\
15 & 2 & 2 & F & 3 & 0 & 1 & 0 & 0 \\
16 & 2 & 3 & M & 3 & 0 & 1 & 0 & 0 \\
17 & 2 & 2 & M & 4 & 0 & 1 & 0 & 0 \\
18 & 2 & 2 & F & 4 & 1 & 0 & 0 & 0 \\
19 & 2 & 3 & F & 4 & 2 & 0 & 0 & 0 \\
20 & 2 & 3 & M & 4 & 0 & 0 & 2 & 0 \\
& & & & & & & & \\
\hline
\end{tabular}

(a) $\mathrm{M}$ : male ; F : female.

(b) [11] : alive, normal ; [12] : alive, assisted ; [21] : dead, normal ; [22] : dead, assisted. 
Bivariate and univariate marginal relative frequencies, by level of each of the 4 factors considered, are presented in table 3 . Overall, about 71 p. 100 of the calves were born alive and $68 \mathrm{p} .100$ of the calvings were normal. Differences among sires for calf livability ranged between 67 and 78 p. 100 ; the corresponding figures for calving difficulty were 33 and 86 p. 100 . When the 2 traits are considered jointly, the data suggest an association between calf mortality and calving difficulty. Given that the calves were alive at birth, the frequency of normal calvings was about 75 p. 100 ; among dead calves, only 50 p. 100 of the calves were normal.

\section{TABLE 3}

Bivariate and univariate marginal raw frequencies for calf viability and calving difficulty by level of the factors considered.

Fréquence marginale de la viabilité du veau et des difficultés de vêlage par niveau de facteur.

\begin{tabular}{|c|c|c|c|c|c|c|c|}
\hline \multirow[b]{2}{*}{ Factor } & \multirow[b]{2}{*}{ Level } & \multicolumn{2}{|c|}{ Alive } & \multicolumn{2}{|c|}{ DEAD } & \multirow[b]{2}{*}{$\begin{array}{c}\text { Alive } \\
{[1-]}\end{array}$} & \multirow[b]{2}{*}{$\begin{array}{c}\text { Normal } \\
{[-1]}\end{array}$} \\
\hline & & $\begin{array}{c}\text { Normal } \\
{[11]}\end{array}$ & $\begin{array}{c}\text { Assisted } \\
{[12]}\end{array}$ & $\begin{array}{c}\text { Normal } \\
{[21]}\end{array}$ & $\begin{array}{c}\text { Assisted } \\
\text { [22] }\end{array}$ & & \\
\hline Herd year & $\begin{array}{l}1 \\
2\end{array}$ & $\begin{array}{l}.5385 \\
.5333\end{array}$ & $\begin{array}{l}.1538 \\
.2000\end{array}$ & $\begin{array}{l}.1538 \\
.1333\end{array}$ & $\begin{array}{l}.1538 \\
.1333\end{array}$ & $\begin{array}{l}.6923 \\
.7333\end{array}$ & $\begin{array}{l}.6923 \\
.6666\end{array}$ \\
\hline Cow Age. & $\begin{array}{l}2 \\
3\end{array}$ & $\begin{array}{l}.6154 \\
.4667\end{array}$ & $\begin{array}{l}.2308 \\
.1333\end{array}$ & $\begin{array}{l}.0000 \\
.2667\end{array}$ & $\begin{array}{l}.1538 \\
.1333\end{array}$ & $\begin{array}{l}.8462 \\
.6000\end{array}$ & $\begin{array}{l}.6154 \\
.7334\end{array}$ \\
\hline Sex of Calf $\ldots \ldots \ldots$ & $\begin{array}{l}\mathrm{M} \\
\mathrm{F}\end{array}$ & $\begin{array}{l}.4286 \\
.6429\end{array}$ & $\begin{array}{l}.2143 \\
.1429\end{array}$ & $\begin{array}{l}.2143 \\
.0714\end{array}$ & $\begin{array}{l}.1429 \\
.1429\end{array}$ & $\begin{array}{l}.6429 \\
.7858\end{array}$ & $\begin{array}{l}.6429 \\
.7143\end{array}$ \\
\hline Sire & $\begin{array}{l}1 \\
2 \\
3 \\
4\end{array}$ & $\begin{array}{l}.7143 \\
.5556 \\
.3333 \\
.5000\end{array}$ & $\begin{array}{l}.0000 \\
.2222 \\
.3333 \\
.1667\end{array}$ & $\begin{array}{l}.1429 \\
.1111 \\
.0000 \\
.3333\end{array}$ & $\begin{array}{l}.1429 \\
.1111 \\
.3333 \\
.0000\end{array}$ & $\begin{array}{l}.7143 \\
.7778 \\
.6666 \\
.6667\end{array}$ & $\begin{array}{l}.8572 \\
.6667 \\
.3333 \\
.8333\end{array}$ \\
\hline$\ldots \ldots \ldots$ & - & .5357 & .1786 & .1429 & .1429 & .7143 & .6786 \\
\hline
\end{tabular}

\section{B. Models}

The same explanatory variables were included in the models for the 2 conceptual variables for calf viability (A) and calving difficulty (B). The models for the parameters in $(12 \mathrm{a})$ and $(12 \mathrm{~b})$ were :

$$
\begin{aligned}
\mu_{j}^{x}=H_{k}^{x}+A_{1}^{x}+S_{m}^{x}+u_{n}^{x} & \\
& x=A, B \\
j & =1, \ldots, 20
\end{aligned}
$$

where $H_{k}^{x}$ is the effect of the $k$ th herd-year $(k=1,2), A_{\ell}^{x}$ is the effect of the $\ell$ th age of dam ( $\ell=$ heifers, cows), $S_{m}^{x}$ is the effect of the $m$ th sex of calf $(m=$ males, females), and $u_{n}^{x}$ is the effect of the $n$th sire of the calf $(n=1 \ldots, 4)$. In order to have 
$\mathbf{X}_{\mathrm{A}}$ and $\mathbf{X}_{\mathrm{B}}$ in (11) with full-column rank, the corresponding $\beta$-vectors were taken as :

$$
\boldsymbol{\beta}_{\mathbf{x}}=\left[\begin{array}{l}
H_{1}^{x}+A_{1}^{x}+S_{1}^{x} \\
H_{2}^{x}+A_{1}^{x}+S_{1}^{x} \\
A_{2}^{x}-A_{1}^{x} \\
S_{2}^{x}-S_{1}^{x}
\end{array}\right] ; x=A, B
$$

The first 2 elements of $\boldsymbol{\beta}_{\mathrm{x}}$ correspond to location parameters for male calves born out of heifers in herd-years 1 and 2, respectively. Likewise, the 3rd and 4th elements of $\boldsymbol{\beta}_{\mathrm{x}}$ represent contrasts between cows and heifers, and female and male calves, respectively.

\section{Prior information}

As pointed out in section III, prior information about $\boldsymbol{\beta}_{\mathrm{A}}$ and $\boldsymbol{\beta}_{\mathrm{B}}$ was assumed to be vague. Now, from (18) and (19):

$$
\mathbf{G}=\left[\begin{array}{ll}
g_{\mathrm{AA}} I & g_{\mathrm{AB}} I \\
g_{\mathrm{AB}} I & g_{\mathrm{BB}} I
\end{array}\right]
$$

where $\mathrm{g}_{\mathrm{AA}}\left(\mathrm{g}_{\mathrm{BB}}\right)$ is the variance among sires for trait $\mathrm{A}(\mathrm{B}), \mathrm{g}_{\mathrm{AB}}$ is the sire component of covariance between traits $A$ and $B$, and $I$ is an identity matrix of order 4 . The inverse of $\mathbf{G}$, required in (29) or (30), is :

$$
\mathbf{G}^{-1}=\left(\frac{1}{1-\mathrm{r}_{\mathrm{G}}^{2}}\right)\left[\begin{array}{lr}
\lambda_{\mathrm{A}} & -\mathrm{r}_{\mathrm{G}} \sqrt{\lambda_{A} \lambda_{B}} \\
-\mathrm{r}_{\mathrm{G}} \sqrt{\lambda_{A} \lambda_{B}} & \lambda_{B}
\end{array}\right] \otimes \mathbf{I}
$$

where :

$\otimes$ is the Kronecker product ;

$r_{G}$ is the genetic correlation between calf mortality and calving difficulty, assumed equal to .70 (PhilipPSON et al., 1979); and

$\lambda_{\mathrm{x}}=\left(4-\mathrm{h}_{\mathrm{x}}^{2}\right) / \mathrm{h}_{\mathrm{x}}^{2}\left(\mathrm{X}=\mathrm{A}, \mathrm{B} ; \mathrm{h}_{\AA}^{2}=.05 ; \mathrm{h}_{\mathrm{B}}^{2}=.20 ;\right.$ PHILIPPSON et al., 1979).

The parameter $\varrho$, required to evaluate $\phi(., ., ; \varrho)$, and $\Phi(., . ; \varrho)$, the probabilities of response (eqs. $10 \mathrm{a}-10 \mathrm{~d}$ ), was assumed to be equal to .40. This correlation can be interpreted as the intra-sire residual correlation between the two conceptual variates.

\section{Iteration}

Equations (29) were used to proceed with iteration. Two sets of starting values were used :

a) the solution to (29) with :

$$
\begin{aligned}
& { }^{*} \mathbf{W}_{\mathrm{AA}}=\mathbf{W}_{\mathrm{BB}}=\operatorname{Diag}\left\{\mathbf{n}_{\mathrm{j}}\right\} ; \mathrm{j}=1, \ldots, 20 \\
& { }^{*} \mathbf{W}_{\mathrm{AB}}=\mathbf{0} \\
& { }^{*} \mathbf{u}_{\mathrm{A}}=\mathbf{u}_{\mathrm{B}}=\mathbf{0}
\end{aligned}
$$




$$
\begin{aligned}
& { }^{*} \mathbf{v}_{\mathrm{A}}=\left\{\mathrm{n}_{\mathrm{j}, 11}+\mathrm{n}_{\mathrm{j}, 12}\right\} ; \mathrm{j}=1, \ldots, 20 \\
& { }^{*} \mathbf{v}_{\mathrm{B}}=\left\{\mathbf{n}_{\mathrm{j}, 11}+\mathrm{n}_{\mathrm{j}, 21}\right\} ; \mathrm{j}=1, \ldots, 20
\end{aligned}
$$

These starting values are, in fact, solutions to univariate " mixed model " equations arising from treating separately each of the 2 binary traits, calf viability and calving difficulty. The variance ratios used were $\lambda_{\mathrm{A}}=79$ and $\lambda_{\mathrm{B}}=19$, which correspond to the heritability values described previously;

b) the solution to (29) with :

$$
\begin{aligned}
& { }^{*} \mathbf{W}_{\mathrm{AA}}=\mathbf{W}_{\mathrm{BB}}=\mathbf{I} \\
& { }^{*} \mathbf{W}_{\mathrm{AB}}=\mathbf{0} \\
& { }^{*} \mathbf{u}_{\mathrm{A}}=\mathbf{u}_{\mathrm{B}}=\mathbf{0} \\
& { }^{*} \mathbf{v}_{\mathrm{A}}=\left\{\ln \left[\frac{\mathbf{n}_{\mathrm{j}, 11}+\mathbf{n}_{\mathrm{j}, 12}+.50}{\mathbf{n}_{\mathrm{j}, 21}+\mathbf{n}_{\mathrm{j}, 22}+.50}\right]\right\} ; \mathrm{j}=1, \ldots, 20 \\
& { }^{*} \mathbf{v}_{\mathrm{B}}=\left\{\ln \left[\frac{\mathbf{n}_{\mathrm{j}, 11}+\mathbf{n}_{\mathrm{j}, 21}+.50}{\mathbf{n}_{\mathrm{j}, 12}+\mathbf{n}_{\mathrm{j}, 22}+.50}\right]\right\} ; \mathrm{j}=1, \ldots, 20
\end{aligned}
$$

The criterion used to stop iterations was :

$$
\varepsilon=\sqrt{\Delta^{\prime} \Delta / 2(p+q)}<10^{-8}
$$

where $\boldsymbol{\Delta}$ is the vector of corrections in (29), $p=$ order of $\boldsymbol{\beta}_{A}=$ order of $\boldsymbol{\beta}_{B}, q=$ order of $\mathbf{u}_{\mathrm{A}}=$ order of $\mathbf{u}_{\mathrm{B}}$. Bivariate normal integrals were computed using formulae described by Ducroco (1984); these are shown in Annex C.

\section{F. Results}

Five iterations were required to satisfy the above stopping rule and convergence to the same solution occurred irrespective of the starting set. The results of iteration using the set (a) of initial values are presented in table 4 . Although the stopping rule was not satisfield until the 5 th round $\left(\varepsilon=.7 \times 10^{-9}\right)$, iteration could have stopped in the 4 th one as the solutions remained virtually unchanged thereafter.

Estimates of the components of $\boldsymbol{\theta}$ and the square root of their estimated posterior variances are presented in table 5 ; posterior dispersion was calculated from elements of the inverse of the coefficient matrix in (29) with $\mathbf{W}_{\mathrm{AA}}, \mathbf{W}_{\mathrm{BB}}$ and $\mathbf{W}_{\mathrm{AB}}$ evaluated after the solutions stabilized. Also shown in table 5 are estimates of $\theta$ obtained from 2 separate univariate evaluations, one for calf viability and the other for calving difficulty. In order to interpret the results, it is convenient to refer to equations $(10 \mathrm{a}-10 \mathrm{~d})$ and (32) and to the way that the data were classified (table 1). Thus, the marginal probabilities of a live birth or of a normal calving for the jth row of the contingency table increase as $\mu_{j}^{\mathrm{A}}$ or $\mu_{j}^{\mathrm{B}}$ increase. In the conceptual scale, the results from the bivariate analysis indicate that female calves had higher viability $(.293 \pm .539)$ and easier births $(.341 \pm .529)$ than male calves. Cows had easier calvings than heifers $(.413 \pm .533)$ but their calves were less viable than those out of heifers $(-.742 \pm .562)$. This surprising result, peculiar to the hypothetical data set used, was also obtained in the univariate 
TABLE 4

Solutions by round of iteration using univariate " mixed model " solutions to $(0,1)$ data as starting values.

Solutions aux différentes itérations en partant de solutions unidimensionnelles des équations du modèle mixte sur données $(0,1)$.

\begin{tabular}{|c|c|c|c|c|c|}
\hline \multirow{2}{*}{ Element } & \multicolumn{5}{|c|}{ ITERATION (a) } \\
\hline & 1 & 2 & 3 & 4 & 5 \\
\hline $\mathbf{H}_{1}^{\mathrm{A}}+\mathbf{A}_{\mathbf{1}}^{\mathbf{A}}+\mathbf{S}_{1}^{\mathrm{A}} \ldots \ldots \ldots \ldots$ & 788.78 & 855.75 & 880.92 & 881.08 & 881.08 \\
\hline $\mathrm{H}_{2}^{\mathrm{A}}+\mathrm{A}_{1}^{\mathrm{A}}+\mathrm{S}_{1}^{\mathrm{A}} \ldots \ldots \ldots \ldots \ldots$ & 791.59 & 866.00 & 889.38 & 889.56 & 889.56 \\
\hline $\mathbf{A}_{2}^{\mathbf{A}}-\mathbf{A}_{1}^{\mathbf{A}} \ldots \ldots \ldots \ldots \ldots$ & -228.41 & -724.01 & -741.42 & -741.60 & -741.60 \\
\hline $\mathbf{S}_{2}^{\mathrm{A}}-\mathrm{S}_{1}^{\mathrm{A}} \ldots \ldots \ldots \ldots \ldots \ldots$ & 91.28 & 287.21 & 292.82 & 292.89 & 292.89 \\
\hline $\mathbf{u}_{1}^{\mathrm{A}} \ldots \ldots \ldots \ldots \ldots \ldots \ldots$ & -4.07 & 29.80 & 30.58 & 30.59 & 30.59 \\
\hline $\mathbf{u}_{2}^{\mathrm{A}} \ldots \ldots \ldots \ldots \ldots \ldots$ & 8.13 & $-\quad 2.62$ & $-\quad 2.65$ & 2.65 & $-\quad 2.65$ \\
\hline $\mathbf{u}_{3}^{\mathrm{A}} \ldots \ldots \ldots \ldots \ldots \ldots$ & 2.77 & -46.16 & -46.54 & -46.55 & -46.55 \\
\hline $\mathbf{u}_{4}^{\mathrm{A}} \ldots \ldots \ldots \ldots \ldots \ldots$ & 1.29 & 18.99 & 18.61 & 18.61 & 18.61 \\
\hline $\mathbf{H}_{1}^{\mathrm{B}}+\mathbf{A}_{1}^{\mathrm{B}}+\mathbf{S}_{1}^{\mathrm{B}} \ldots \ldots \ldots \ldots \ldots$ & 575.15 & 123.68 & 134.54 & 134.55 & 134.55 \\
\hline $\mathbf{H}_{2}^{\mathrm{B}}+\mathbf{A}_{1}^{\mathrm{B}}+\mathbf{S}_{1}^{\mathbf{B}} \ldots \ldots \ldots \ldots$ & 527.55 & 40.16 & 48.48 & 48.49 & 48.48 \\
\hline $\mathbf{A}_{2}^{\mathbf{B}}-\mathbf{A}_{1}^{\mathbf{B}} \ldots \ldots \ldots \ldots$ & 144.52 & 418.82 & 413.00 & 412.99 & 412.99 \\
\hline $\mathbf{S}_{2}^{\mathrm{B}}-\mathbf{S}_{1}^{\mathrm{B}} \ldots \ldots \ldots \ldots \ldots$ & 102.66 & 343.96 & 340.67 & 340.67 & 340.67 \\
\hline $\mathbf{u}_{1}^{\mathrm{B}} \ldots \ldots \ldots \ldots \ldots \ldots$ & 60.28 & 102.27 & 104.89 & 104.89 & 104.89 \\
\hline $\mathbf{u}_{2}^{\mathrm{B}} \ldots \ldots \ldots \ldots \ldots \ldots$ & -18.45 & -28.42 & -28.97 & -28.97 & -28.97 \\
\hline $\mathbf{u}_{3}^{\mathrm{B}} \ldots \ldots \ldots \ldots \ldots \ldots$ & -79.78 & -140.58 & -141.84 & -141.85 & -141.85 \\
\hline 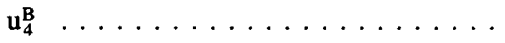 & 37.95 & 66.72 & 65.72 & 65.93 & 65.93 \\
\hline
\end{tabular}

(a) Entries should be multiplied by $10^{-3}$

analysis. Sire rankings for the conceptual variable for calving ease were the same in the univariate and bivariate analyses. This did not happen in the case of calf viability, where sire solutions were strongly pulled towards zero because of low heritability.

As pointed out by Gianola \& Foulley (1983) and Foulley et al. (1983), it may be of interest in animal selection to rank sires on the basis of response probabilities rather than on values of conceptual variables measured in residual standard deviation units. Given a category c $(11,12,21,22)$ one would calculate for each sire (in the context of eq. 32) :

$$
\hat{\Pi}_{\mathrm{n}, \mathrm{c}}=\sum_{\mathrm{k}} \sum_{\ell} \sum_{\mathrm{m}} \mathrm{a}_{\mathrm{k} \ell \mathrm{m}} \hat{\mathrm{P}}_{\mathrm{k \ell mn}, \mathrm{c}} ; \mathrm{n}=1, \ldots, 4
$$

where $\hat{\mathrm{P}}_{\mathrm{k} \ell \mathrm{mn}, \mathrm{c}}$ is either $(10 \mathrm{a}),(10 \mathrm{~b}),(10 \mathrm{c})$ or $(10 \mathrm{~d})$ evaluated at arguments appropriate for the $\mathrm{k} \ell \mathrm{mn}$ th subclass, and $\mathrm{a}_{\mathrm{k} \ell \mathrm{m}}$ is a weight such that $0 \leq \mathrm{a}_{\mathrm{k} \ell \mathrm{m}} \leq 1$ and $\sum_{\mathrm{k}} \sum_{\ell} \sum_{\mathrm{m}} \mathrm{a}_{\mathrm{k} \ell \mathrm{m}}=1$. 
TABLE 5

Estimates of parameters and of their posterior precision for univariate and bivariate models.

Estimation des paramètres et de leur précision

a posteriori pour des modeles uni et bidimensionnels.

\begin{tabular}{|c|c|c|c|}
\hline \multirow{2}{*}{ TRAIT } & \multirow{2}{*}{ PARAMETER } & \multicolumn{2}{|c|}{ MODEL } \\
\hline & & UNIVARIATE & BIVARIATE \\
\hline Calf Viability & 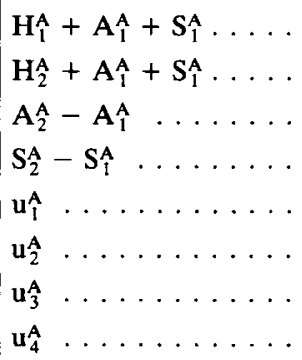 & $\begin{aligned} .835 & \pm .606 \\
.861 & \pm .566 \\
-.711 & \pm .550 \\
.293 & \pm .532 \\
-.004 & \pm .111 \\
.012 & \pm .111 \\
-.005 & \pm .111 \\
-.002 & \pm .111\end{aligned}$ & $\begin{aligned} .881 & \pm .626 \\
.890 & \pm .576 \\
-.742 & \pm .562 \\
.293 & \pm .539 \\
.031 & \pm .109 \\
-.003 & \pm .108 \\
-.047 & \pm .108 \\
.019 & \pm .109\end{aligned}$ \\
\hline Calving Ease & 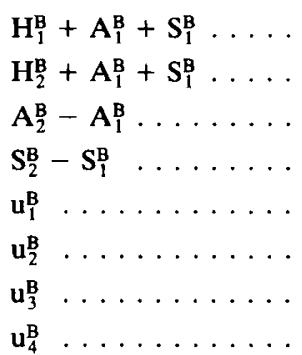 & $\begin{array}{r}.162 \pm .580 \\
.063 \pm .535 \\
.410 \pm .526 \\
.307 \pm .523 \\
.108 \pm .215 \\
-.033 \pm .214 \\
-.145 \pm .215 \\
.070 \pm .219\end{array}$ & $\begin{array}{r}.135 \pm .593 \\
.048 \pm .538 \\
.413 \pm .533 \\
.341 \pm .529 \\
.105 \pm .215 \\
-.029 \pm .215 \\
-.142 \pm 215 \\
.066 \pm .219\end{array}$ \\
\hline
\end{tabular}

In order to illustrate, sires were evaluated for heifer calvings equally distributed over herd-years and sexes. Thus, from (10 a) :

$$
\begin{gathered}
\hat{\Pi}_{n, 11}=\frac{1}{4}\left[\sum_{k=1}^{2} \sum_{m=1}^{2} \Phi\left(\hat{H}_{k}^{A}+\hat{A}_{l}^{A}+\hat{S}_{m}^{A}+\hat{\mathbf{u}}_{n}^{A}, \hat{H}_{k}^{B}+\hat{A}_{1}^{B}+\hat{S}_{m}^{B}+\hat{\mathbf{u}}_{n}^{B} ; .40\right)\right] \\
n=1, \ldots, 4
\end{gathered}
$$

where $a_{\mathrm{k} \ell \mathrm{m}}=0$ if $\ell=2$ (cows) and $1 / 4$ otherwise. The results are presented in table 6 in terms of joint and marginal probabilities in the bivariate analysis, and of marginal (and joint, assuming independence) probabilities in the univariate approach. While estimates of marginal probabilities of response were similar in univariate and bivariate analyses, this was not always the case for joint probabilities. For example, the univariate analysis gave estimates for the [22] category (dead calf, difficult calving) which were considerably lower than those obtained from the bivariate approach. If rankings were to be based on joint probabilities, the 2 analyses would have rankek the sires equally, irrespective of the category. However, if sires are ranked on the basis of the marginal probability of calf livability, the univariate procedure would give $2>1=3>4$, and the bivariate one would yield $1>4>2>3$. Nevertheless, differences among sires for this trait cannot be considered of practical importance, at least in this data set. 
TABLE 6

Marginal and joint weighted (a) probabilities of response in heifer calvings, by sire.

Probabilité marginale de réponse par père en vêlage de génisses.

\begin{tabular}{l|c|c|c|c|c}
\hline \multirow{2}{*}{ Model } & Category ${ }^{(b)}$ & \multicolumn{4}{|c}{ SIRE } \\
\cline { 2 - 5 } & & 1 & 2 & 3 & 4 \\
\hline \multirow{3}{*}{ Univariate (c) $^{*}$} & {$[1-]$} & & & & \\
& {$[-1]$} & .836 & .840 & .836 & .837 \\
& {$[1-] \times[-1]$} & .644 & .591 & .547 & .630 \\
& {$[1-] \times[-2]$} & .538 & .496 & .457 & .527 \\
& {$[2-] \times[-1]$} & .298 & .344 & .379 & .310 \\
& {$[2-] \times[-2]$} & .106 & .095 & .090 & .103 \\
& {$[1-]$} & .058 & .065 & .074 & .060 \\
\hline \multirow{2}{*}{ Bivariate } & {$[-1]$} & .853 & .846 & .835 & .851 \\
& {$[11]$} & .641 & .591 & .547 & .627 \\
& {$[12]$} & .585 & .540 & .499 & .572 \\
& {$[21]$} & .268 & .306 & .337 & .279 \\
& {$[22]$} & .056 & .051 & .048 & .054 \\
& & .091 & .103 & .116 & .095 \\
\hline
\end{tabular}

(a) Weighted equally across herd-years and sexes. See eq. (38).

(b) See table 3 .

(c) Joint probabilities in the univariate case calculated as if the 2 traits were independent.

\section{Discussion}

This study is in the context of an effort to develop a unified methodology for the analysis of categorical data in animal breeding (GiANOLA \& FoulleY, 1982, 1983 ; FOULLEY et al., 1983). The general strategy consists of postulating conceptual normal variates following a mixed linear model, and mapping these variates onto a discrete scale via hypothetical fixed thresholds, usually unknown. The location parameters of the underlying distribution are then estimated in a Bayesian framework as the mode of a joint posterior density. Advantages and limitations of the methodology and its relationship to other approaches have been described in preceeding articles (e.g., GIANOLA \& FOULLEY, 1983) so these matters will not be dealt with here. The study further illustrates the generality of the principles used by extending the method to include bivariate binary categorical responses.

While this paper focuses on bivariate " all or none" responses, the approach can be extended, without formal difficulty, to multiple binary or polychotomous responses. For reasons of space, this will be addressed in a future communication. Bivariate models have been already proposed by NERLOVE \& PRESs (1973) but their method used logistic functions and was restricted to fixed effects. Application of the method to more than 
2 or 3 binary responses, as in THOMPSON (1972) dealing with a related problem, probably raises important numerical issues related either to the difficulty of evaluating with sufficient precision multivariate normal integrals, or to the size of the system requiring solution in the course of iteration.

It is pertinent to address the question of how much efficiency is gained by using a multiple trait nonlinear evaluation procedure as opposed to a univariate one. The latter evaluation requires knowing heritability in the conceptual scale and it has been demonstrated (MEIJERING \& Gianola, unpubl. results) that sire rankings are quite insensitive to errors in this parameter. A multivariate evaluation requires, in addition, knowledge of genetic correlations between traits and this can be a limiting factor in many situations. Hence, multiple trait evaluations are not always justifiable from a practical viewpoint, particularly when the benefits are contrasted with the high costs of computer implementation.

The procedure described here assumes that the 2 binary responses are scored in every individual or experimental unit. However, it would be possible to adapt the method to the case of incomplete information along the lines of the procedures described by Foulley et al. (1982) for continuous responses. Such an adaptation would make the method more general, and perhaps more appealing, for application to populations undergoing selection.

\section{Acknowledgements}

The Holstein Association, Brattleboro, Vermont, U.S.A., is acknowledged for supporting research on categorial data being undertaken at the University of Illinois. J.J. COLLEAU from I.N.R.A., Jouy-en-Josas is thanked for providing formulae and routines for the calculation of normal integrals. One of the anonymous referees is thanked for a suggestion leading to formula (30) which shows more clearly the analogy betwen the multiple trait mixed model equations and the proposed methology.

Received December 6, 1983.

Accepted March 20, 1984.

\section{References}

Abramowitz M., Stegun A., 1972. Handbook of mathematical functions, Dover Publications Inc., 1045 pp., New York.

Bоoтн A.D., 1957. Numerical methods. Butterworths Scientific Publications, London, 195 p.

Dahlouist G., Bjorck A., 1974. Numerical methods. Prentice Hall, Englewood Cliffs.

Ducroco V., 1984. Conséquences sur le progrès génétique laitier d'une sélection sur des caractères secondaires chez les bovins. Génét. Sél. Evol. (submitted).

DuTt J.E., Soms A.P., 1976. An integral representation technique for calculating general multivariate probabilities with and application to multivariate $\chi^{2}$. Commun. Stat. Theor. Method, A 5, 3777-3788.

Foulley J.L., Calomiti S., Gianola D., 1982. Ecriture des équations du BLUP multicaractères. Ann. Génét. Sél. Anim., 14, 309-326. 
Foulley J.L., Gianola D., Thompson R., 1983. Prediction of genetic merit from data on categorical and quantitative variates with an application to calving difficulty, birth weight and pelvic opening. Génét. Sél. Evol., 15, 407-424.

Gianola D., 1982. Theory and analysis of threshold characters. J. Anim. Sci., 54, 1079-1096.

Gianola D., Foulley J.L., 1982. Non-linear prediction of latent genetic liability with binary expression : an empirical Bayesian approach. Second World Congress on Genetics Applied to Livestock Production, Madrid, 4-8 october 1982, 7, 293-303, Garsi, Madrid.

Gianola D., Foulley J.L., 1983. Sire evaluation for ordered categorical data with a threshold model. Génét. Sél. Evol., 15, 201-223.

Goffinet B., 1983. Selection on selected records. Génét. Sél. Evol., 15, 91-97.

Gurland J., 1948. Inversion formulae for the distribution of ratios. Ann. Math. Stat., 19, 228-237.

Henderson C.R., 1973. Sire evaluation and genetic trends. Proceedings of the Animal Breeding and Genetics Symposium in Honor of Dr Jay L. Lush, Blacksburg, Virginia, July 29, 1982, American Society of Animal Science and American Dairy Science Association, 10-41, Champaign, Illinois.

Henderson C.R., QuAas R.L., 1976. Multiple trait evaluation using relatives' records. J. Anim. Sci., 43, 1188-1197.

Kendall M.G., Stuart A., 1945. The advanced theory of statistics. Vol. 1, 433 pp., Griffin, London.

LINDLEY D.V., 1965. Introduction to probability and statistics from a Bayesian viewpoint. Part 2. Inference, 292 pp., Cambridge University Press, Cambridge.

Mardia K.V., Kent J.T., BibBy J.M., 1979. Multivariate analysis, 521 pp., Academic Press, London.

Mineur H., 1966. Techniques de calcul numérique, 605 pp., Dunod, Paris.

Nerlove M., Press S.J., 1973. Univariate and multivariate log-linear and logistic models. Rand Corporation, R-1306-EDA/NIH. Santa Monica, California.

Philipsson J., Foulley J.L., Lederer J., Liboriussen T., OsingA A., 1979. Sire evaluation standards and breeding strategies for limiting dystocia and stillbirth. Livest. Prod. Sci., 6, 111-127.

Pratt J.W., Raiffa H., SChlaifer R., 1965. Introduction to statistical decision theory. Mc GrawHill, New York.

QuAAS R.L., VAN VLECK L.D., 1980. Categorical trait sire evaluation by best linear unbiased prediction of future progeny category frequencies. Biometrics, 36, 117-122.

SChaEffer L.R., Wilton J.W., 1976. Methods of sire evaluation for calving ease. J. Dairy Sci., 59, 544-551.

TALlis G.M., 1962. The maximum likelihood estimation of correlation from contingency tables. Biometrics, 18, 342-353.

Thompson R., 1972. The maximum likelihood approach to the estimate of liability. Ann. Hum. Genet., 36, 221-231. 


\section{Annex A}

First derivatives of the log-posterior with respect to the parameters : Some usefull results From (9), neglecting suffixes :

$$
\begin{aligned}
\mathrm{P}_{11} & =\int_{-\infty}^{\mu^{\mathrm{A}}} \int_{-\infty}^{\mu^{\mathrm{B}}} \phi\left(\mathrm{y}^{*} \mid \mathrm{x}^{*}\right) \cdot \phi\left(\mathrm{x}^{*}\right) \mathrm{dx}^{*} \mathrm{dy}^{*} \\
& =\int_{-\infty}^{\mu^{\mathrm{A}}} \phi\left(\mathrm{x}^{*}\right) \Phi\left(\frac{\mu^{\mathrm{B}}-\varrho \mathrm{x}^{*}}{\sqrt{1-\mathrm{Q}^{2}}}\right) d \mathrm{x}^{*}
\end{aligned}
$$

where $\phi($.$) and \Phi($.$) are univariate normal density and distribution functions. Alterna-$ tively :

$$
\mathrm{P}_{11}=\int_{-\infty}^{\mu_{\mathrm{B}}} \phi\left(\mathrm{y}^{*}\right) \Phi\left(\frac{\mu^{\mathrm{A}}-\varrho \mathrm{y}^{*}}{\sqrt{1-\mathrm{Q}^{2}}}\right) d \mathrm{y}^{*}
$$

From (A 1) :

$$
\frac{\partial \mathrm{P}_{11}}{\partial \mu^{\mathrm{A}}}=\phi\left(\mu^{\mathrm{A}}\right) \Phi\left(\frac{\mu^{\mathrm{B}}-\mathrm{Q} \mu^{\mathrm{A}}}{\sqrt{1-\mathrm{Q}^{2}}}\right)=\phi\left(\mu^{\mathrm{A}}\right) \Phi\left(\mathrm{h}_{\mathrm{B}}\right)
$$

where $h_{B}=\left(\mu^{B}-\varrho \mu^{A}\right) / \sqrt{1-\varrho^{2}}$. Using (A 3) in (10 b) - (10 d), one obtains :

$$
\begin{aligned}
& \frac{\partial \mathrm{P}_{12}}{\partial \mu^{\mathrm{A}}}=\phi\left(\mu^{\mathrm{A}}\right)\left[1-\Phi\left(\mathrm{h}_{\mathrm{B}}\right)\right] \\
& \frac{\partial \mathrm{P}_{21}}{\partial \mu^{\mathrm{A}}}=-\phi\left(\mu^{\mathrm{A}}\right) \Phi\left(\mathrm{h}_{\mathrm{B}}\right) \\
& \frac{\partial \mathrm{P}_{22}}{\partial \mu^{\mathrm{A}}}=-\phi\left(\mu^{\mathrm{A}}\right)\left[1-\Phi\left(\mathrm{h}_{\mathrm{B}}\right)\right]
\end{aligned}
$$

Similarly :

$$
\begin{aligned}
\frac{\partial P_{11}}{\partial \mu^{B}} & =\phi\left(\mu^{B}\right) \Phi\left(h_{A}\right) \\
\frac{\partial P_{12}}{\partial \mu^{B}} & =-\phi\left(\mu^{B}\right) \Phi\left(h_{A}\right) \\
\frac{\partial P_{21}}{\partial \mu^{B}} & =\phi\left(\mu^{B}\right)\left[1-\Phi\left(h_{A}\right)\right] \\
\frac{\partial P_{22}}{\partial \mu^{B}} & =\phi\left(\mu^{B}\right)\left[1-\Phi\left(h_{A}\right)\right] \\
\text { where } h_{A} & =\left(\mu^{A}-\varrho \mu^{B}\right) / \sqrt{1-Q^{2}} .
\end{aligned}
$$

First derivatives with respect to $\beta_{A}, \beta_{B}, \mathbf{u}_{A}$ and $\mathbf{u}_{B}$ 
From (24) and (A 3) - (A 6), one obtains after algebra :

$$
\begin{aligned}
\frac{\partial \mathbf{L}(\boldsymbol{\theta})}{\partial \boldsymbol{\beta}_{\mathrm{A}}} & =\sum_{\mathrm{j}} \sum_{\mathbf{k}} \mathbf{n}_{\mathrm{j}, \mathrm{k}} \frac{\partial \ell \mathrm{n} \mathbf{P}_{\mathrm{j}, \mathbf{k}}}{\partial \mu^{\mathrm{A}}} \cdot \frac{\partial \mu^{\mathrm{A}}}{\partial \boldsymbol{\beta}_{\mathrm{A}}} \\
& =\sum_{\mathrm{j}} \mathbf{v}_{\mathrm{jA}} \mathbf{x}_{\mathrm{jA}}=\mathbf{X}_{\mathrm{A}}^{\prime} \mathbf{v}_{\mathrm{A}}
\end{aligned}
$$

where :

$\mathbf{v}_{\mathrm{A}}=\left\{\mathrm{v}_{\mathrm{jA}}\right\}, \mathrm{j}=1 \ldots, \mathrm{s}$, and :

$$
\begin{aligned}
& v_{j A}=\phi\left(\mu_{j}^{A}\right) \Phi\left(h_{j B}\right)\left(\frac{n_{j, 11}}{P_{j, 11}}-\frac{n_{j, 21}}{P_{j, 21}}\right) \\
& +\phi\left(\mu_{j}^{A}\right)\left[1-\Phi\left(h_{j B}\right)\right]\left(\frac{n_{j, 12}}{P_{j, 12}}-\frac{n_{j, 22}}{P_{j, 22}}\right)
\end{aligned}
$$

Similarly :

$$
\frac{\partial \mathrm{L}(\boldsymbol{\theta})}{\partial \boldsymbol{\beta}_{\mathrm{B}}}=\mathbf{X}_{\mathrm{B}}^{\prime} \mathbf{v}_{\mathrm{B}}
$$

where :

$\mathbf{v}_{\mathrm{B}}=\left\{\mathbf{v}_{\mathrm{jB}}\right\}, \mathrm{j}=1 \ldots, \mathrm{s}$, and :

$$
\begin{aligned}
& v_{j B}=\phi\left(\mu_{j}^{B}\right) \Phi\left(h_{j A}\right)\left(\frac{n_{j, 11}}{P_{j, 11}}-\frac{n_{j, 12}}{P_{j, 12}}\right) \\
& +\phi\left(\mu_{j}^{B}\right)\left[1-\Phi\left(h_{j A}\right)\right]\left(\frac{n_{j, 21}}{P_{j, 21}}-\frac{n_{j, 22}}{P_{j, 22}}\right)
\end{aligned}
$$

Formulae (A 12) and (A 14) can be obtained from the general expression :

$$
\mathrm{v}_{\mathrm{j} \ell}=\sum_{\mathrm{r}_{1}} \sum_{\mathrm{r}_{2}} \frac{\mathrm{n}_{\mathrm{j}, \mathrm{r}_{1} \mathrm{r}_{2}}}{\mathrm{P}_{\mathrm{j}, \mathrm{r}_{1} \mathrm{r}_{2}}}(-1)^{\mathrm{r}_{\ell}-1} \phi\left(\mu_{\mathrm{j} \ell}\right) \Phi\left[(-1)^{\mathrm{r}_{\ell^{\prime}}-1} \mathrm{~h}_{\mathrm{j} \ell^{\prime}}\right]
$$

where $\left[\ell=1(\mathrm{~A}), \ell^{\prime}=2(\mathrm{~B})\right]$, or $\left[\ell=2(\mathrm{~B}), \ell^{\prime}=1(\mathrm{~A})\right]$, and $\mathrm{r}_{1}, \mathrm{r}_{2}$ indicate the category of response; for example, a response in [21] would be indicated as $r_{1}=2 \cdot r_{2}=1$. Note that when $\varrho=0$ :

$$
\begin{aligned}
& \mathrm{P}_{11}=\Phi\left(\mu^{\mathrm{A}}\right) \Phi\left(\mu^{\mathrm{B}}\right) ; \mathrm{P}_{12}=\Phi\left(\mu^{\mathrm{A}}\right)\left[\left(1-\Phi\left(\mu^{\mathrm{B}}\right)\right]\right. \\
& \mathrm{P}_{21}=\left[1-\Phi\left(\mu^{\mathrm{A}}\right)\right] \Phi\left(\mu^{\mathrm{B}}\right) ; \mathrm{P}_{22}=\left[1-\Phi\left(\mu^{\mathrm{A}}\right)\right]\left[1-\Phi\left(\mu^{\mathrm{B}}\right)\right]
\end{aligned}
$$

and (A 12) reduces to :

$$
v_{j A}=n_{j, 1} \frac{\phi\left(\mu^{A}\right)}{\Phi\left(\mu^{A}\right)}-n_{j, 2 .} \frac{\phi\left(\mu^{A}\right)}{\left[1-\Phi\left(\mu^{A}\right)\right]}
$$

which is the formula corresponding to the univariate case with binary responses (GIANOLA \& Foulley, 1983). 
From (24), the first derivatives with respect to $\mathbf{u}_{\mathrm{A}}$ and $\mathbf{u}_{\mathrm{B}}$ include a contribution from the prior density. Thus :

$$
\frac{\partial \mathbf{L}(\boldsymbol{\theta})}{\partial \mathbf{u}_{\mathrm{A}}}=\mathbf{Z}_{\mathrm{A}}^{\prime} \mathbf{v}_{\mathrm{A}}-\mathbf{G}^{\mathrm{AA}} \mathbf{u}_{\mathrm{A}}-\mathbf{G}^{\mathrm{AB}} \mathbf{u}_{\mathrm{B}}
$$

and :

$$
\frac{\partial \mathbf{L}(\boldsymbol{\theta})}{\partial \mathbf{u}_{\mathrm{B}}}=\mathbf{Z}_{\mathrm{B}}^{\prime} \mathbf{v}_{\mathrm{B}}-\mathbf{G}^{\mathrm{BA}} \mathbf{u}_{\mathrm{A}}-\mathbf{G}^{\mathrm{BB}} \mathbf{u}_{\mathrm{B}}
$$

\section{Annex B}

Second derivatives of the log-posterior with respect to the parameters

From (A 11) :

$$
\begin{aligned}
\frac{\partial^{2} \mathbf{L}(\boldsymbol{\theta})}{\partial \boldsymbol{\beta}_{\mathrm{A}} \partial \boldsymbol{\beta}_{\mathrm{A}}^{\prime}} & =\mathbf{X}_{\mathrm{A}}^{\prime}\left\{\frac{\partial v_{\mathrm{jA}}}{\partial \mu_{\mathrm{j}}^{\mathrm{A}}} \cdot \frac{\partial \mu_{\mathrm{j}}^{\mathrm{A}}}{\partial \boldsymbol{\beta}_{\mathrm{A}}^{\prime}}\right\}=\mathbf{X}_{\mathrm{A}}^{\prime}\left\{\frac{\partial v_{\mathrm{jA}}}{\partial \mu_{\mathrm{j}}^{\mathrm{A}}} \mathbf{x}_{\mathrm{jA}}^{\prime}\right\} \\
& =-\mathbf{X}_{\mathrm{A}}^{\prime} \operatorname{diag}\left\{-\frac{\partial \mathbf{v}_{\mathrm{jA}}}{\partial \mu_{\mathrm{j}}^{\mathrm{A}}}\right\} \mathbf{X}_{\mathrm{A}}=-\mathbf{X}_{\mathrm{A}}^{\prime} \mathbf{W}_{\mathrm{AA}} \mathbf{X}_{\mathrm{A}}
\end{aligned}
$$

as in (28 a). Similarly, in $(28 \mathrm{~b})$ and $(28 \mathrm{c})$ :

$$
\begin{aligned}
& \mathbf{W}_{\mathrm{BB}}=\operatorname{Diag}\left\{-\frac{\partial \mathbf{v}_{\mathrm{jB}}}{\partial \mu_{\mathrm{j}}^{\mathrm{B}}}\right\} \\
& \mathbf{W}_{\mathrm{AB}}=\operatorname{Diag}\left\{-\frac{\partial \mathbf{v}_{\mathrm{iA}}}{\partial \mu_{\mathrm{j}}^{\mathrm{B}}}\right\}
\end{aligned}
$$

The derivation of individual elements of (B 2) and (B 3) requires tedious algebra which will not be presented here. For example, writing $\mu_{j}^{A}=\mu_{j A}, \mu_{j}^{B}=\mu_{j B}$ :

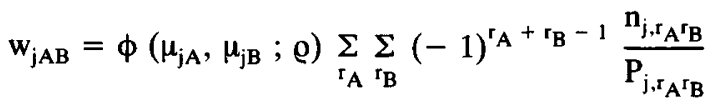

$$
\begin{aligned}
& -\phi\left(\mu_{j A}, \mu_{j B} ; 0\right) \sum_{r_{A}} \sum_{r_{B}}(-1)^{r_{A}+r_{B}-1} \Phi\left[(-1)^{r_{A}-1} h_{j A}\right] \Phi\left[(-1)^{r_{B}-1} h_{j B}\right] \frac{n_{j, r_{A} r_{B}}}{P_{j, r_{A} r_{B}}}
\end{aligned}
$$

with $\phi(., .$, ; $)$ the standard bivariate normal density function.

With the above results, the remaining second derivatives needed in $(28 \mathrm{a})-(28 \mathrm{j})$ can be obtained in a straightforward manner. Observe that in $(28 \mathrm{~h})-(28 \mathrm{j})$, the prior density contributes to second derivatives with respect to $\mathbf{u}_{\mathrm{A}}$ and $\mathbf{u}_{\mathrm{B}}$. 


\section{Annex C}

\section{Evaluation of bivariate normal integrals}

DUTT \& SOMS (1976) described a technique for calculating multivariate normal probabilities. Because their general expressions and notation are complex, only the principles used and the results applicable to univariate and bivariate integrals are presented.

\section{General principles}

1) Integrals of $n$ dimensions, each evaluated between a threshold and infinity, are transformed into a sum of $2^{n}$ integrals of dimensions 1 to $n$, between $-\infty$ and $\infty$. This permits integration using known numerical methods.

Changes in integration limits are accomplished writing the probability density function entering the integrand as an integral (between $-\infty$ and $\infty$ ) of the characteristic function, and then using the "inversion theorem" (Kendall \& STUART, 1945 ; MARDIA et al., 1979). The characteristic function is then fragmented into lower dimension characteristic functions which correspond to the marginal distributions (GURLAND, 1948).

2) The elementary integrals so obtained are then calculated numerically using the " quadratization" method of Gauss. The simplest integrals, of the form :

$$
\int_{-\infty}^{+\infty} \exp \left(-\frac{x^{2}}{2}\right) g(x) d x
$$

are approximated by :

$$
\sum_{i=1}^{k} w_{i} g\left(h_{i}\right)
$$

where the $h_{i}(i=1, \ldots, k)$ are the $k$ positive roots of Hermite polynomials of order $2 k$, orthogonal to $\exp \left(-\frac{x^{2}}{2}\right)$, and the $w_{i}$ 's are weights calculated as a function of $h_{i}$ and of $\exp \left(-\frac{x^{2}}{2}\right)$ (BоOTH, 1957 ; Mineur, 1966). Multiple integrals can be brought to the form (C 1) by successive " quadratization".

\section{Application to univariate and bivariate integrals}

We used the highest polynomial with already calculated roots i.e. $2 \mathrm{k}=20$ (ABRAMOWITZ \& STEGUN, 1972) :

$$
I_{1}=\int_{a}^{+\infty} \phi(x) d x
$$

was approximated as :

$$
\hat{\mathrm{I}}_{1}=.5-\frac{1}{\Pi} \sum_{\mathrm{i}=1}^{10} \frac{\mathrm{w}_{\mathrm{i}}}{\mathrm{h}_{\mathrm{i}}} \sin \left(\mathrm{ah}_{\mathrm{i}}\right)
$$


In the case of bivariate volumes :

$$
\mathrm{I}_{2}=\int_{a}^{+\infty} \int_{b}^{+\infty} \phi(x, y ; \varrho) d x d y
$$

was approximated as :

$$
\begin{gathered}
\hat{l}_{2}=.25-\frac{1}{2 \Pi} \sum_{i=1}^{10} \frac{w_{i}}{h_{i}}\left[\sin \left(a h_{i}\right)+\sin \left(b h_{i}\right)\right] \\
+\frac{1}{2 \Pi^{2}} \sum_{i=1}^{10} \sum_{i=1}^{10} \frac{w_{i}}{h_{i}} \frac{w_{j}}{h_{j}} \cos \left(a h_{i}-b h_{j}\right)[\exp (\rho a b)-\exp (-\rho a b)]
\end{gathered}
$$

